\title{
WW domain binding protein 5 induces multidrug resistance of small cell lung cancer under the regulation of miR-335 through the Hippo pathway
}

\author{
Ruixiang Tang ${ }^{1,3}$, Yingying Lei ${ }^{1,2,3}$, Bingshuang $\mathrm{Hu}^{1}$, Jie Yang ${ }^{1}$, Shun Fang ${ }^{1}$, Qiongyao Wang ${ }^{1}$, Man $\mathrm{Li}^{1}$ \\ and Linlang Guo*,1 \\ ${ }^{1}$ Department of Pathology, Zhujiang Hospital, Southern Medical University, Guangzhou, China and 2Department of Oncology, \\ Panyu Maternal and Child Care Service Centre of Guangzhou \& Hexian Memorial affiliated hospital of Southern Medical \\ University, Guangzhou, China
}

Background: Our previous study indicated that WW domain binding protein 5 (WBP5) expression was elevated significantly in a drug-resistant cell compared with its parental cell. Nevertheless, its functional role and underlying mechanisms remain unknown.

\begin{abstract}
Methods: In this study, WBP5 was examined in 62 small cell lung cancer (SCLC) patient samples by immunohistochemical technique. Stable WBP5-overexpressed and WBP5-underexpressed cells were further established to assess the role of WBP5 in drug resistance, apoptosis and tumour growth. We also conducted western blot to detect the expression of MST2 and YAP1 and their phosphorylated protein.
\end{abstract}

Results: The results revealed that WBP5 expression was significantly associated with the shorter survival time in SCLC patients. Upregulation of WBP5 induced multidrug resistance (MDR) and decreased apoptosis, whereas downregulation of WBP5 enhanced drug sensitivity and increased apoptosis. We also found that miR-335 negatively regulated the MDR of WBP5 by targeting its 3'UTR. Furthermore, WBP5 can lower YAP1 phosphorylation at Serine 127 and induce nuclear accumulation of YAP1. Inhibition of YAP1 by Verteporfin could blunt the MDR phenotype of WBP5.

Conclusions: WW domain binding protein 5 can modulate MDR through the Hippo pathway under the regulation of miR-335. WW domain binding protein 5 may be a prognostic predictor and a potential target for interfering with MDR in SCLC.

Small cell lung cancer (SCLC) is the most aggressive form of lung cancer, accounting for approximately $13 \%$ of all lung cancers, which is characterised by short doubling time, fast growth speed, highly invasive ability and distant metastasis (Reinke et al, 2016). Although presenting a better initial reactivity to the front-line standard chemotherapy regimen (etoposide or irinotecan plus cisplatin), SCLC recurs shortly after the first successful treatment with multidrug resistance (MDR) phenotype, and the 2-year survival rate is less than 5\% (Metro et al, 2012; Nagy-Mignotte et al, 2012). Hence, the understanding of the mechanisms contributing to chemotherapy drug resistance, particularly MDR, will be paramount to the treatment of SCLC.

WW domains are typically 35-40 amino acids in length that interact with a variety of distinct peptide ligands, including motifs with core proline-rich sequences (Bork and Sudol, 1994; Sudol et al, 1995a,b). WW domain binding protein 5 (WBP5)

\footnotetext{
*Correspondence: Professor L Guo; E-mail:linlangg@yahoo.com

${ }^{3}$ These authors contributed equally to this work.
}

Received 29 February 2016; revised 9 May 2016; accepted 12 May 2016; published online 23 June 2016

(c) 2016 Cancer Research UK. All rights reserved 0007-0920/16 
was first identified as one of eight ligands (WBP3 through WBP10) that can bind to FBP11 WW domain in a mouse limb bud expression library (Bedford et al, 1997). WW domain binding protein 5 can also bind to Abl, which may be a novel upstream activator of MST2 in the Hippo pathway (Liu et al, 2012). Despite some studies showed that WBP5 is lower in advanced gastric cancer with aggressive lymph node metastasis than that in advanced tumour stage but without lymph node metastasis (Suh et al, 2015) and that WBP5 is one of the 15 candidate oncogenes in human colorectal cancers with microsatellite instability (Gylfe et al, 2013), little is known about WBP5, especially in SCLC.

MicroRNAs (miRNAs) are a class of conserved small noncoding RNAs of 18-24 nucleotides. They have important roles in cell growth, differentiation and apoptosis through regulating translation and stability of their target mRNAs mainly by binding to the $3^{\prime}$-untranslated region ( $3^{\prime}$-UTR) of their target mRNAs (Lan et al, 2015). Recently, some studies also suggested that miRNAs are closely related to chemotherapy resistance (Hummel et al, 2010; Bai et al, 2014; Zhang et al, 2015). MiR-335 was reported to act as a novel tumour suppressor to regulate clear cell renal cell carcinoma cell proliferation and invasion through downregulation of BCL-W expression (Wang et al, 2015). MiR-335 may confer sensitivity to anti-cancer drugs by targeting SIAH2 (Kim et al, 2015). Loss of miR-335 promoted SCLC metastatic skeletal lesions via deregulation of IGF-IR and RANKL pathways (Gong et al, 2014). However, there is still no report available about miR-335 functions in SCLC drug resistance until now.

The Hippo pathway was originally identified as the signalling that controls organ size in Drosophila, with the core architecture conserved in mammals. The central components of the mammalian Hippo pathway include MST1/2 (mammalian Ste20-like kinases), LATS1/2 (large tumour suppressor kinases), YAP1 (transcriptional co-activators, Yes-associated protein) and TAZ (Transcriptional co-activator with a PDZ-binding motif) (Kodaka and Hata, 2015). YAP1/TAZ can shuttle between the cytoplasm and the nucleus. YAP1/TAZ can induce the expression of cellproliferative and anti-apoptotic genes via interactions with transcription factors particularly TEA domain family members (TEADs) in the nucleus (Mo et al, 2014; Moroishi et al, 2015). When the inhibitory Hippo kinase module is 'on', MST1/2 phosphorylate LATS1/2, and then LATS1/2 phosphorylate YAP1/ TAZ. Once YAP1 is phosphorylated by LATS1/2 at serine 127 , YAP1 is sequestrated in the cytoplasm because of binding to the 14-3-3 protein, and the output gene production (e.g., CTGF, AREG,Gli2 and so on) (Zhao et al, 2008, 2010; Haskins et al, 2014) is therefore 'turned off (Basu et al, 2003). In contrast, when the Hippo pathway is 'off, YAP1/TAZ translocate into the nucleus (Johnson and Halder, 2014). In non-SCLC, YAP1 protein acts as a transcriptional co-activator of Oct4. The physical interaction between YAP1 and Oct4 is necessary for self-renewal and vascular mimicry of stem-like cells (Bora-Singhal et al, 2015). YAP1 may have an important role in the prognosis of small-cell lung cancer patients treated with platinum-based chemotherapy, as YAP1 variants are associated with survival of small-cell lung cancer patients (Wu et al, 2010). Nevertheless, the role of Hippo pathway in SCLC remains to be fully elucidated.

Our previous study indicated that WBP5 expression was significantly elevated in drug-resistant cell compared with its parental cell (Guo et al, 2010). To assess the biological role of WBP5 in SCLC, we examined WBP5 expression in 62 SCLC patient samples. We also evaluated the relevance of WBP5 expression to clinical prognosis of SCLC. Then, we investigated the role of WBP5 in MDR in vitro and in vivo. We also confirmed the interaction between WBP5 and miR-335 by luciferase reporter assays. Furthermore, we revealed that the MDR phenotype of WBP5 was modulated by the Hippo pathway.

\section{MATERIALS AND METHODS}

Patient samples. Sixty-two SCLC patient samples were collected from patients who had received bronchofiberscopy or biopsy between January 2008 and March 2012. They all received care and follow-up in Southern Medical University affiliated Zhujiang Hospital (Guangzhou). Under the protocol approved by the Institutional Review Board, informed consent was obtained from the patients or their guardians. The Ethical Committee of Zhujiang Hospital approved the tissue collection and studies with collected tumour tissues. Clinical characteristics included the patient's age, sex and disease stage, and follow-up is collected and summarised.

Immunohistochemical staining. Immunohistochemical staining was undertaken using the En Vision two-step method (DAKO, Glostrup, Denmark) following the manufacturer's instruction. The formalin-fixed, paraffin-embedded tissues were sectioned and deparaffinised and rehydrated routinely. The slides were then incubated with a primary antibody, directed against WBP5 (Santa Cruz, Dallas, TX, USA; $1: 100$ dilution), at $4{ }^{\circ} \mathrm{C}$ overnight. The biotinylated secondary antibody (1:500 dilution; Dako, Carpinteria, CA, USA) and the avidin-biotin complex (Vector Labs, Burlingame, CA, USA) were then applied according to the manufacturer's recommendations.

All slides were assessed by two pathologists. The results were classified semiquantitatively based on the total scores of the percent positivity of stained tumour cells and the staining intensity. The percent positivity was scored as 0 if $<5 \%$ (negative), 1 if $5-30 \%$ (sporadic), 2 if $30-70 \%$ (focal) and 3 if $>70 \%$ (diffuse) of cells stained, whereas staining intensity was scored relative to the known positive and negative controls as 0 if no staining, 1 if weakly to moderately stained and 2 if strongly stained (Takahashi et al, 2009). The score $\geqslant 2$ is regarded as 'High' and the score $<2$ is regarded as 'Low' in immunohistochemical staining.

Cell culture. The human small lung cancer cell lines NCI-H69, NCI-H446 and the drug-resistant subline H69AR were obtained from the ATCC. The adriamycin resistant subline H446AR was obtained by our team as previously described (Bai et al, 2014). All cells were maintained in RPMI1640 (Hyclone, Logan City, UT, USA) with 10\% FBS (Gibco, Logan City, UT, USA) and grown in a humidified atmosphere of $5 \% \mathrm{CO}_{2}$ and $95 \%$ air.

Transfection. For miRNA and siRNA transient transfection, cells were placed in standard media for $24 \mathrm{~h}$ before being transfected with miR-335 mimics, inhibitors, miRNA negative control (miRNC) and siMST2 (GenePharma, Shanghai, China) using Lipofectamine 2000 and OPTI-MEMI (Invitrogen, Carlsbad, CA, USA) according to the manufacturer's protocol.

For stable transfection, pGPU6/GFP/Neo-shWBP5 (GenePharma) was transfected into H69AR and H446AR cells by Lipofectamine 2000 (Invitrogen), a nonsilencing plasmid pGPU6/GFP/Neo-shNC was used as control; $\mathrm{H} 69$ and $\mathrm{H} 446$ cells were transfected with PEX2-WBP5 or PEX2 empty vector (GenePharma) using Lipofectamine 2000 according to the manufacturer's instructions. Positive transfectants were selected with G418 (H69AR, $300 \mu \mathrm{g} \mathrm{ml}^{-1}$; H446AR, $500 \mu \mathrm{g} \mathrm{ml}^{-1}$; H69, $200 \mu \mathrm{g} \mathrm{ml}^{-1}$; H446, $300 \mu \mathrm{g} \mathrm{ml}^{-1}$ ) in 1 month. G418-resistant cells were ring-cloned and expanded to establish stable transfectants (H69-WBP5, H69-NC, H69AR-shWBP5, H69AR-shNC, H446-WBP5, H446-NC, H446ARshWBP5, H446AR-shNC). Infection efficiency was confirmed by quantitative RT-PCR (qRT-PCR) and western blot.

RNA isolation, reverse transcription and quantitative real-time PCR. Total RNA, including miRNA, from sorted cells was extracted using RNeasy kits (Qiagen, Dusseldorf, Germany) or the miRNeasy kit (Qiagen) and reverse-transcribed into cDNA 
using Tiangen reverse transcription reagents (Tiangen, Beijing, China) according to the manufacturer's instructions.

The quantitative PCR reaction was carried out in a ABI Illumina Instrument (Foster City, CA, USA) using SYBR Green (Tiangen). The miRNA sequence-specific reverse transcription qRT-PCR for miR-335 and endogenous control U6 were performed using the Hairpin-it miRNAs qRT-PCR quantisation kit and U6 snRNA real-time PCR normalisation kit (GenePharma, Shanghai, China). GAPDH or U6 snRNA was used as an endogenous control.

All samples were normalised to the internal controls, and fold changes were calculated through relative quantification $\left(2^{-\Delta \Delta \mathrm{Ct}}\right)$.

Western blot. Total protein was extracted from cells using RIPA lysis buffer (Sigma-Aldrich, Shanghai, China) and equilibrated using Pierce BCA protein assay reagent (Logan City, UT, USA). Lysates were resolved on 10\% SDS-PAGE gels and electroblotted onto PVDF membrane (Bio-Rad, Hercules, CA, USA). After the PVDF membranes were blocked with 5\% BSA, they were incubated with primary antibodies (WBP5, Santa Cruz, 1:500 dilution; MST2, Abcam (Cambridge, UK), 1:500 dilution; phospho-MST1 (Thr183)/MST2 (Thr180), Cell Signaling Technology (Shanghai, China), 1:500 dilution; YAP1, Abcam, 1:1000 dilution; YAP1 (phosphor S127), Abcam, 1:500 dilution; GAPDH, Abcam, $1: 10000$ dilution), followed by the appropriate peroxidase-linked secondary rabbit anti-goat IgG antibody or goat anti-mouse IgG or goat anti-rabbit IgG antibody. GAPDH was used as control. The immune complexes were detected by chemiluminescence.

Colony-forming assay. Cells were plated in six-well culture plates at various cell densities (50-200 cells per well), then cultured for an additional 14 days, and the surviving colonies (a colony was defined as $>50$ cells) were counted.

Cell counting kit-8 (CCK-8) assay. Transient or stable transfected cells were cultured at $5 \times 10^{3}$ cells per well in triplicate in 96-well plates. Following a 6-h culture, the cells were treated with drugs (Adriamycin (ADM; Yifei Biotech, Jiangsu, China), Cisplatin (cDDP; qilu pharma, Shandong, China), Etoposide (VP-16; Yifei Biotech)) for $24 \mathrm{~h}$. After incubation with $10 \mu \mathrm{l}$ of CCK-8 reagent (Dojindo, Kumamoto, Japan) for about $4 \mathrm{~h}$, the absorbance at $450 \mathrm{~nm}$ was measured. Absorbance values were expressed as percentages relative to the controls; $\mathrm{IC}_{50}$ was calculated according to the percentages. Each experiment was performed in triplicate and then averaged.
Flow cytometric analysis. Cells were treated with drugs for $10 \mathrm{~h}$ and then washed twice in cold PBS and fixed with $70 \%$ ethanol. Apoptosis was detected by Annexin V-fluorescein isothiocyanate according to the manufacturer's protocol. The samples were all analysed by flow cytometry (FACS Calibur, Becton Dickinson, Franklin Lakes, NJ, USA) using CellQuest software (BD Bioscience, San Jose, CA, USA).

Luciferase reporter assays. The full-length $3^{\prime}$ UTRs and coding sequences or mutated untranslated regions sequences of WBP5 were cloned into the psiCheck2 dual luciferase reporter vector (Promega, Madison, WI, USA), and then co-transfected with miR-335 mimic or antagomir into H69AR cells. Cells were harvested for luciferase activity assays $48 \mathrm{~h}$ after transfection. A luciferase assay kit (Promega) was used according to the manufacturer's protocol.

In vivo chemosensitivity experiments. Male $\mathrm{BALB} / \mathrm{c}$ nude mice aged 4-6 weeks were purchased from Guangdong Medical Laboratory Animal Center (Foshan, Guangdong, China); all procedures were performed according to the guidelines of the Association for the Assessment and Accreditation of Laboratory Animal Care International. The mice were randomly separated into four groups. Exponentially growing SCLC cells (H69-WBP5, H69-NC, H69AR-shWBP5, H69AR-shNC) were injected subcutaneously into the flanks of mice $\left(5 \times 10^{6}\right.$ cells in $100 \mathrm{ul}$ of PBS per animal). Tumour volume was calculated using the formula $\mathrm{V}=\left(\mathrm{L} \times \mathrm{W}^{2}\right) \times 1 / 2$, where $\mathrm{V}=$ volume, $\mathrm{L}=$ length, $\mathrm{W}=$ width. Tumour size was monitored every 3 days.

When the tumours had reached an average volume of about $150 \mathrm{~mm}^{3}$, the mice were treated with Adriamycin (ADM) $\left(2 \mathrm{mg} \mathrm{kg}^{-1}\right.$, every 3 days), cisplatin (cDDP) $\left(2 \mathrm{mg} \mathrm{kg}^{-1}\right.$, every week), etoposide (VP16) (5 $\mathrm{mg} \mathrm{kg}^{-1}$, every 3 days) (Hillgenberg et al, 1999; Nemati et al, 2000; Jafri et al, 2010). The relative tumour volume $\left(\mathrm{V} / \mathrm{V}_{0}\right)$ was calculated for each animal (ratio of the tumour volume (V) compared with the tumour volume at the beginning of administration $\left(\mathrm{V}_{0}\right)$ ). The mice were euthanised 18 days after administration.

Immunofluorescence. Cells were seeded into 24 -well plates $12 \mathrm{~h}$ before being fixed with $4 \%$ paraformaldehyde. Then, the fixed cells were solubilised in PBS-0.1\% Triton-X100 for $20 \mathrm{~min}$ at room temperature. After washing by PBS three times, cells were incubated with $5 \%$ normal calf serum for $30 \mathrm{~min}$, then incubated with rabbit anti-human YAP1 monoclonal antibody (Abcam, $1: 200$ dilution) at $4{ }^{\circ} \mathrm{C}$ overnight. The cells were stained with conjugated secondary antibody $(1: 100)$ for $1.5 \mathrm{~h}$ at RT in PBS-1\% BSA in the
A

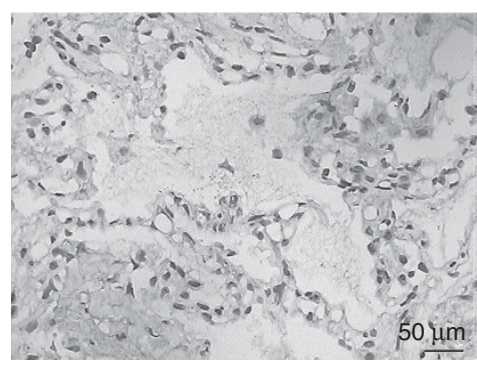

B

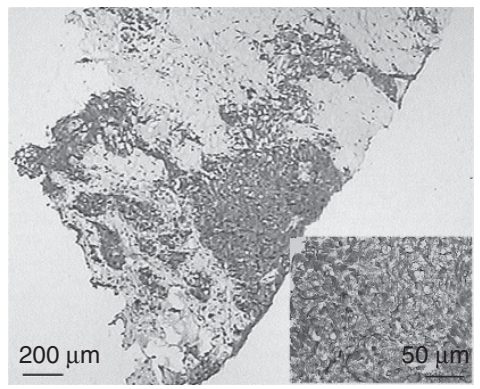

C

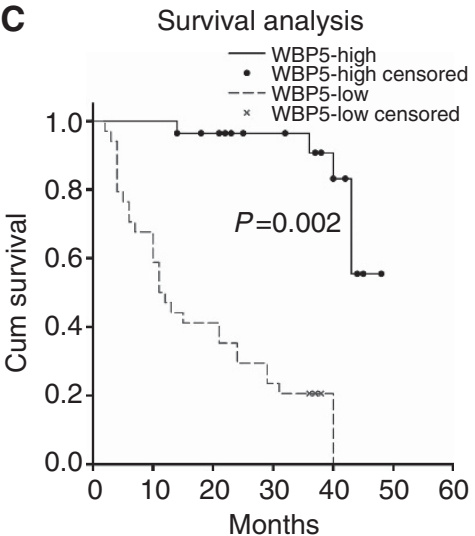

Figure 1. Prognostic analysis for WBP5 performed on clinical samples. (A) Expression of WBP5 in normal lung alveolar epithelium (magnification $\times$ 400). (B) Representative slides showing WBP5 cytoplasm staining in SCLC biopsy samples (left panel: magnification $\times 100$; right panel: magnification $\times 400$ ). (C) Survival differences between the group WBP5 high expression and the group WBP5 low expression were statistically assessed by the Kaplan-Meier method and log-rank test. 
dark at room temperature. Then, capture images using a fluorescence microscope (model Eclipse 660, Nikon, Tokyo, Japan).

Statistical analysis. All experiments were run in triplicate. Quantitative data are presented as means \pm standard deviation (s.d.). All statistical analyses were carried out with SPSS19.0 software. $P$ values were calculated by applying the either an analysis of variance or Student's $t$ test to data from independent experiments. The multiple comparisons were carried out by Dunnett- $t$ (Homogeneity of variance). The association between WBP5 expression and clinical features were analysed by Fisher's exact test. Survival curves were examined by the Kaplan-Meier method. Death as a result of SCLC was the primary end point. Prognostic factors were obtained by Cox hazards model. A difference was considered statistically significant when the $P<0.05$.

\section{RESULTS}

WBP5 expression is correlated with clinical stage and survival in SCLC patients. To investigate the clinical features of WBP5 expression in human SCLC, we performed immunohistochemical
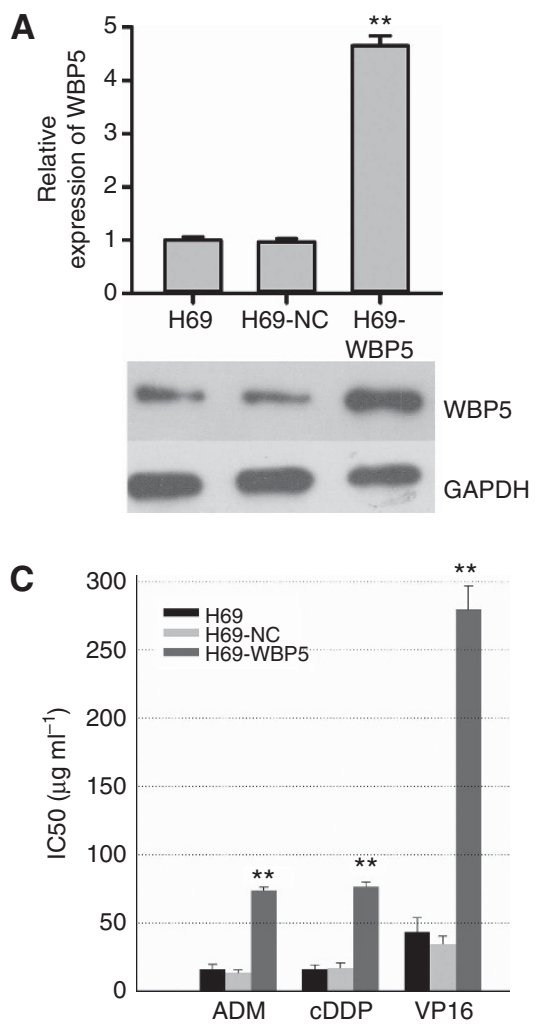

E

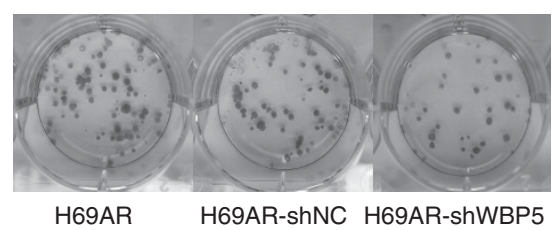

staining to examine WBP5 protein expression in 62 cases of SCLC samples. WW domain binding protein 5 immunoreactivity was predominantly detected in the cytoplasm of cancer cells (Figure $1 \mathrm{~A}$ and $\mathrm{B}$ ). The positive rate of WBP5 expression was $54.8 .5 \%$ (34 out of 62) in SCLC (Supplementary Table S1). WW domain binding protein 5 expression was significantly more frequent at the extensive disease stage (17 out of 19) than at the limited disease stage (17 out of 43) (Supplementary Table S1). Correlation analysis showed that WBP5 expression was significantly associated with clinical stage of SCLC patients $(P=0.000)$, but not with gender $(P=0.306)$ (Supplementary Table $S 1)$. The Kaplan-Meier method revealed that WBP5 expression is correlated with the shorter survival time of the SCLC patients (Figure 1C) $(P=0.002)$.

In addition, we conducted Cox regression analysis using age, sex, stage and WBP5 expression as input variables. The result showed that WBP5 was an independent predictor of survival with a hazard ratio of 8.012 and a $95 \%$ confidence interval ranging from 2.107 to 29.588 .

These findings suggest that expression of WBP5 is associated with clinical prognosis, and WBP5 may be an independent predictive indicator of SCLC.
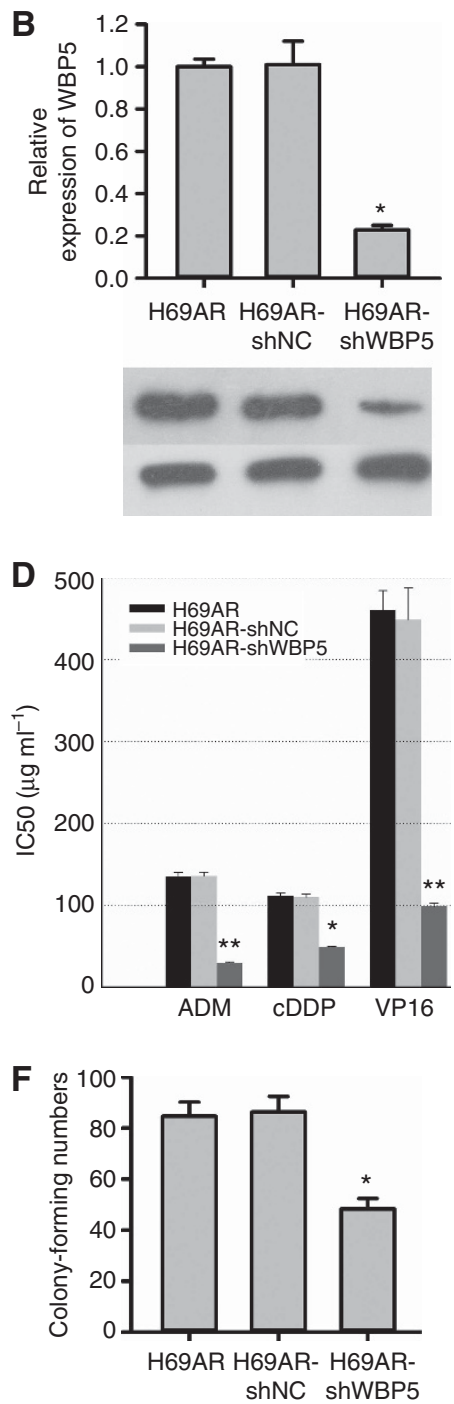

Figure 2. WBP5 can induce MDR and affect clonogenic growth ability of SCLC. (A) WBP5 expression was higher in stable upregulated cell H69WBP5 both in mRNA (upper panel) and protein level (low panel). (B) WBP5 expression was lower in stable downregulated cell H69AR-shWBP5 both in mRNA (upper panel) and protein level (low panel). (C) Upregulation of WBP5 could induce MDR using CCK-8. (D) Downregulation of WBP5 could enhance drug sensitivity of SCLC. (E) Representative pictures showed that downregulation of WBP5 could reduce clonogenic growth ability of SCLC. (F) Bar chart showed that downregulation of WBP5 could reduce clonogenic growth ability of SCLC. ${ }^{\star} P<0.05$, ${ }^{\star *} P<0.01$, compared with control. 
WBP5 expression is associated with SCLC MDR. Our previous data showed that WBP5 expression was 9.67-fold elevated in the H69AR cells as compared with the parental H69 cells using cDNA expression profile analysis (Guo et al, 2010). We then confirmed the WBP5 expression in mRNA and protein level in two pairs of drug-resistant cells (H69AR and H446AR) and their parental cells (H69 and H446) by qRT-PCR and western blot (Supplementary Figure S1A). Using CCK-8 assay, it was demonstrated that H69AR and H446AR cells showed a significantly increased resistance to ADM, cDDP or VP-16 compared with $\mathrm{H} 69$ and H446 (Supplementary Figure S1B).

To investigate the biological roles of WBP5 in human SCLC, we developed WBP5 overexpressed cells by transfecting PEX2-WBP5 plasmid into H69 and H446 cells. We also downregulated WBP5 in H69AR or H446AR cells using pGPU6/GFP/Neo-shWBP5 (Figure 2A and B; Supplementary Figure S2A and B).

Upregulation of WBP5 in $\mathrm{H} 69$ and $\mathrm{H} 446$ resulted in a significant increase of $\mathrm{IC}_{50}$ values after being treated with $\mathrm{ADM}$, cDDP or VP-16 (Figure 2C; Supplementary Figure S2C). In contrast, the $\mathrm{IC}_{50}$ values of WBP5-inhibited transfected cells significantly decreased (Figure 2D; Supplementary Figure S2D).
The effect of WBP5 on cell proliferation was assessed by plate clone forming experiment. The results showed that the number of colonies decreased significantly in H69AR-shWBP5 (Figure 2E and F) compared with control cells. A similar result was observed in H446-WBP5 (Supplementary Figure S2E and F).

Cell apoptosis was determined by flow cytometric analysis after drug exposure. Apoptosis rates significantly decreased in WBP5 upregulated cells, while markedly increased in WBP5 downregulated cells after ADM (Supplementary Figure S3), cDDP (Supplementary Figure S4) and VP16 treatment (Supplementary Figure S5).

Those observations suggested that WBP5 may induce MDR by promoting cell proliferation and inhibiting cell apoptosis of SCLC.

Regulation of MDR by WBP5 is partly subject to miR-335. Our previous study revealed a 7.28-fold decrease of miR-335 expression in H69AR cells compared with $\mathrm{H} 69$ cells by miRNA microarray analysis (Guo et al, 2010)and was verified by qRT-PCR (Figure 3A).

To investigate the effect of miR-335 on MDR in SCLC cells, we transfected SCLC cells with the miR-335 mimics, inhibitor and their corresponding $\mathrm{NC}$, and the infection efficiency was
A
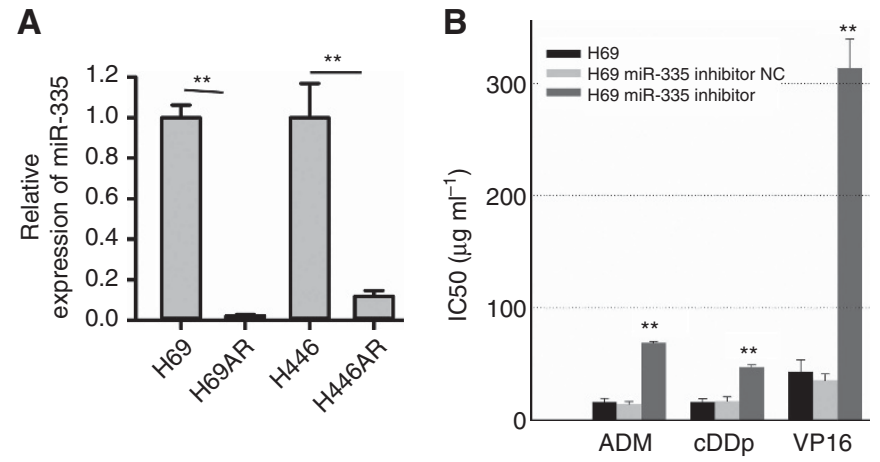

D
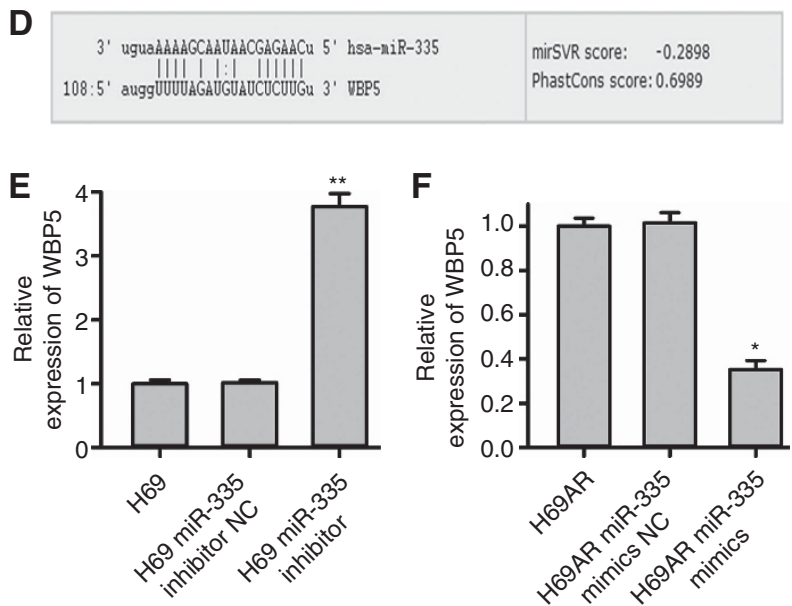

$\mathbf{F}$
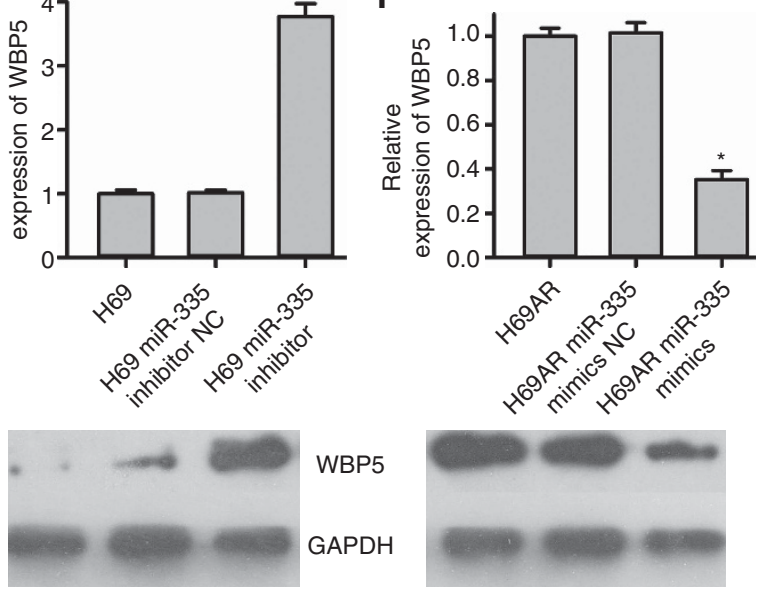

G

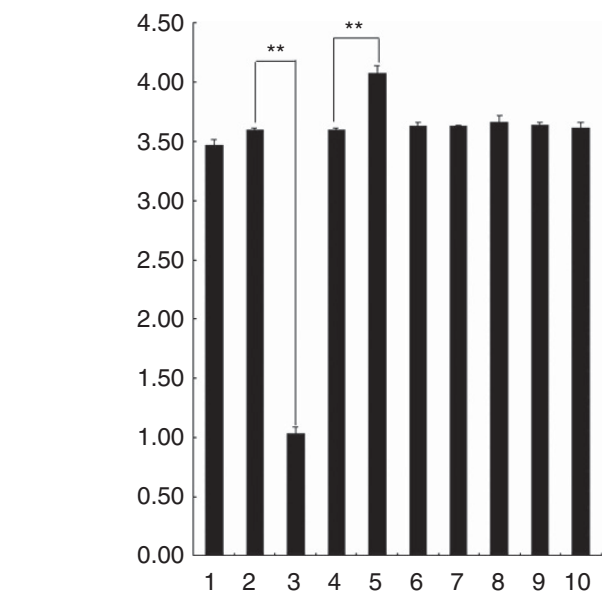

Figure 3. MiR-335 can regulate the MDR of WBP5 by targeting its 3' UTR. (A) qRT-PCR showed that miR-335 is significantly lower in drug-resistant cells than that in their parent cells $(P<0.01)$. (B) The IC 50 of $\mathrm{H} 69$ increased significantly $(P<0.01)$ when miR-335 was inhibited by miR-335 inhibitor; miR-335 inhibitor NC was used as negative control. (C) The $I_{50}$ of H69AR reduced significantly $(P<0.01)$ when miR-335 was upregulated by miR-335 mimics; miR-335 mimics NC was used as negative control. (D) Prediction algorithm predicted miR-335 has potential interaction sites with WBP5 mRNA. (E) miR-335 inhibitor could upregulate WBP5 in mRNA level and protein level. (F) miR-335 mimics could downregulate WBP5.

(G) The dual luciferase assay indicated that miR-335 can directly target WBP5 by binding its $3^{\prime}$-untranslated region. ${ }^{\star} P<0.05$, ${ }^{\star \star} P<0.01$, compared with control. 
confirmed by qRT-PCR (Supplementary Figure S6A, Ba and Ca). The $\mathrm{IC}_{50}$ were increased significantly when the expression of miR-335 was suppressed by miR-335 inhibitor (Figure 3B; Supplementary Figure $\mathrm{S} 6 \mathrm{Bb}$ ), whereas $\mathrm{IC}_{50}$ values were significantly decreased after transfecting with miR-335 mimics (Figure 3C; Supplementary Figure S6Cb).

By searching three prediction algorithms (PicTar, TarScan and miRBase database), we found that miR-335 has potential interaction sites in the $3^{\prime} 3^{\prime} \mathrm{UTR}$ of WBP5 mRNA (Figure 3D). To detect whether WBP5 is subject to miR-335, we analysed the expression of WBP5 by qRT-PCR and western blot when miR-335 is up-regulated or down-regulated. The results showed that WBP5 increased significantly when miR-335 was suppressed by miR-335 inhibitor (Figure 3E; Supplementary Figure S6Bc), whereas WBP5 decreased markedly when miR-335 was upregulated (Figure 3F; Supplementary Figure S6Cc).

To verify whether WBP5 is a direct target of miR-335, we constructed luciferase reporters with mutated (psiCHECK2WBP5-mut-3'UTR) and wild type (psiCHECK2-WBP5 - $3^{\prime}$ UTR) of $3^{\prime}$-UTR of WBP5. Both the wild-type and the mutant-type reporters were introduced into H69AR cells and co-transfected with miR-335 mimics or inhibitors. The luciferase activity of WBP5 was measured by the dual luciferase assays. Compared with the miR-335 NC, miR-335 mimics significantly inhibited $3^{\prime} \mathrm{UTR}$ WBP5 activity and miR-335 inhibitor significantly increased the activity (Figure 3G).

These results suggest that miR-335 may be responsible for the effect of WBP5 on chemosensitivity of SCLC.
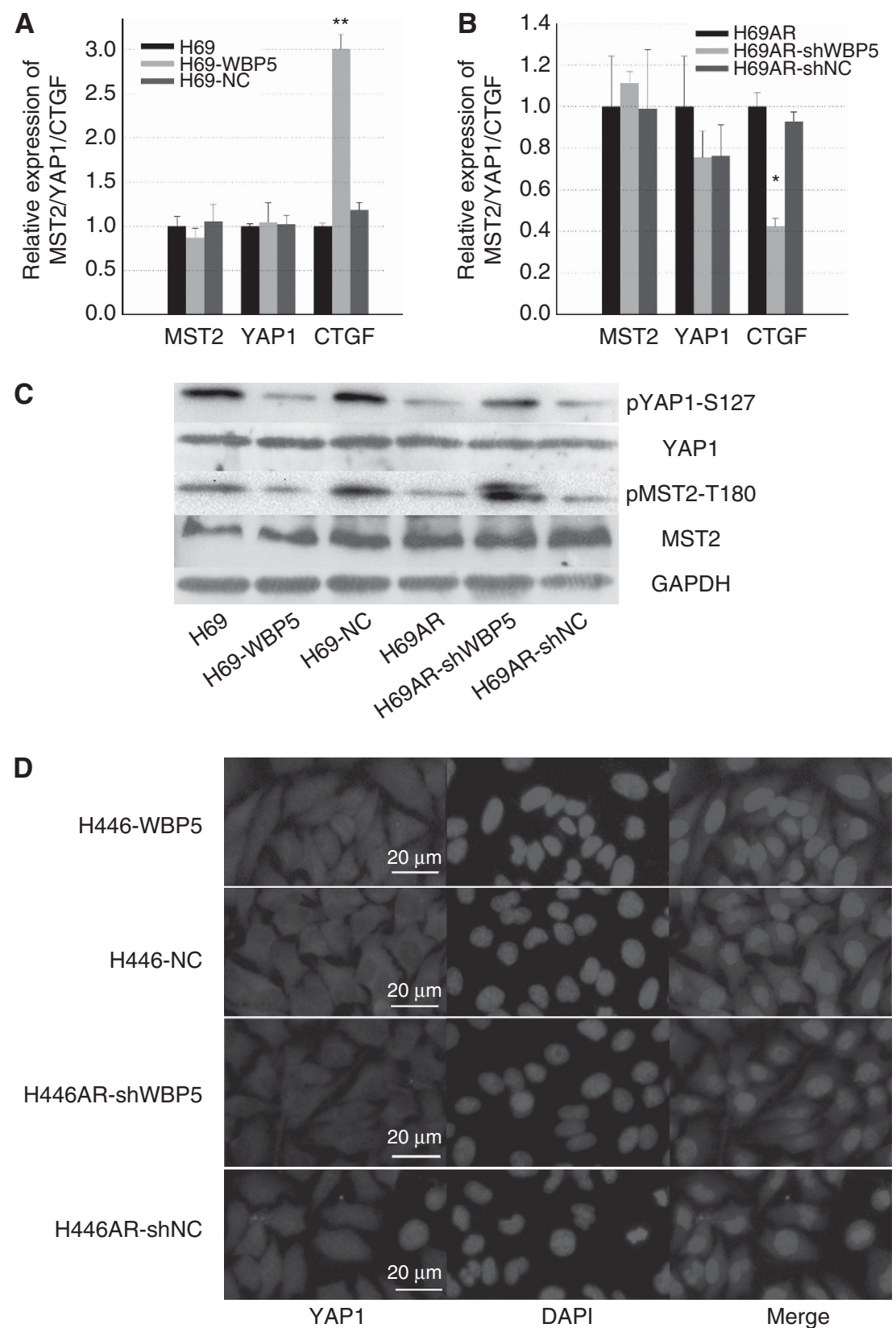

Figure 4. WBP5 may induce SCLC MDR through the Hippo pathway. (A) qRT-PCR showed that upregulation of WBP5 could increase the expression of CTGF. (B) While downregulation of WBP5 could reduce the expression of CTGF, the expression of WBP5 and YAP1 /MST2 was independent. (C) Western lot showed that upregulation of WBP5 could reduce the phosphorylation of pMST2-T180, thus reducing the phosphorylation of PYAP1-S127; downregulation of WBP5 could increase the phosphorylation of pMST2-T180, thus increasing the phosphorylation of pYAP1-S127. (D) The nucleus location of YAP1 was increased in H446-WBP5 compared with H446-NC; the cytoplasm location of YAP1 was decreased in H446AR-shWBP5 compared with H446AR-shNC (magnification $\times 400$ ). ${ }^{\star} P<0.05$, ${ }^{\star \star} P<0.01$, compared with control. 
WBP5 may induce SCLC MDR through the Hippo pathway. WBP5 has been reported to bind to Abl, which may be a novel upstream activator of MST2 in the Hippo pathway (Bedford et al, 1997; Liu et al, 2012). We hypothesised that WBP5 may be involved in SCLC MDR through the WBP5-Abl-MST2-YAP1 pathway (Figure 5A).

CTGF is a downstream gene of the Hippo pathway (Zhao et al, 2010). To assess whether WBP5 is involved in the Hippo pathway, we then conducted qRT-PCR. We found that WBP5 is positively correlated with CTGF, whereas WBP5 showed no correlation with MST2 and YAP1 (Figure 4A and B; Supplementary Figure S7A and B). These findings suggested that WBP5 may affect the Hippo pathway but not on the mRNA level of MST2 and YAP1.

Whether WBP5 affects the Hippo pathway at the protein level, we then conducted western blot to detect the expression of MST2 and YAP1 and their phosphorylated protein when WBP5 was upregulated or downregulated. The results showed that upregulation of WBP5 did not change the expression of MST2 and YAP1, but inhibited the phosphorylation of MST2 (pMST2-T180) and YAP1 (pYAP1-S127). A similar result was observed when WBP5 was downregulated (Figure 4C; Supplementary Figure S7C).
Furthermore, we performed immunofluorescence to observe YAP1 localisation in SCLC cell lines. The result showed that upregulation of WBP5 induced the nuclear accumulation of YAP1. In contrast, downregulation of WBP5 increased the sequestration of YAP1 in cytoplasm (Figure 4D).

Then, we performed gain-of-function and loss-of-function studies to further assess whether the role of WBP5 in MDR is mediated by the Hippo pathway. In WBP5 upregulated cells, we inhibited the activation of YAP1 by Verteporfin (VP) (LiuChittenden et al, 2012) and found that $\mathrm{IC}_{50}$ values decreased significantly compared with control (Figure 5C; Supplementary Figure S8B). Whereas in WBP5 downregulated cells, YAP1 was activated by knocking down MST2 (Figure 5B; Supplementary Figure S8A) and the drug sensitivity was turned over (Figure 5D; Supplementary Figure S8C). Those results showed that WBP5 may induce SCLC MDR through the Hippo pathway.

WBP5 promotes the resistance of SCLC cells to drugs in vivo. To substantiate the MDR effect of $\mathrm{WBP} 5$, in vivo studies were conducted in tumour xenograft models. Four exponentially growing SCLC cells (69-WBP5, 69-NC, 69AR-shWBP5,
A

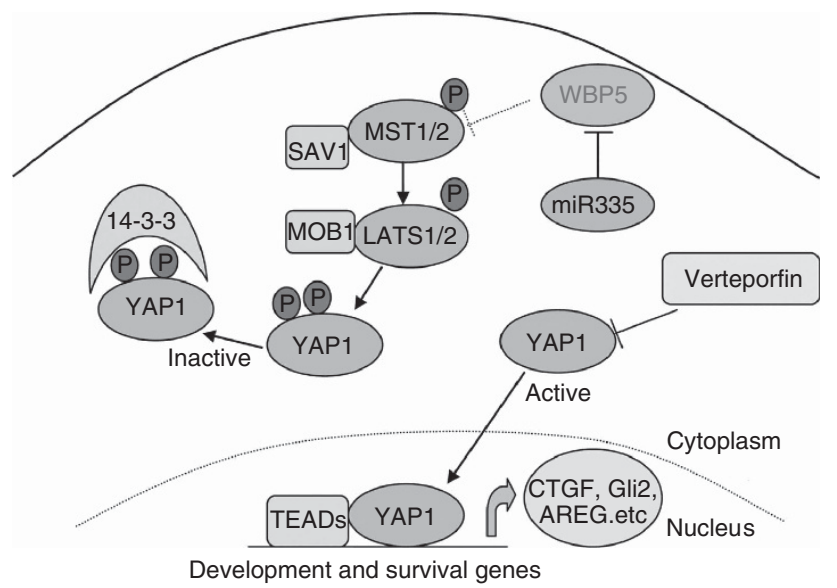

Development and survival genes

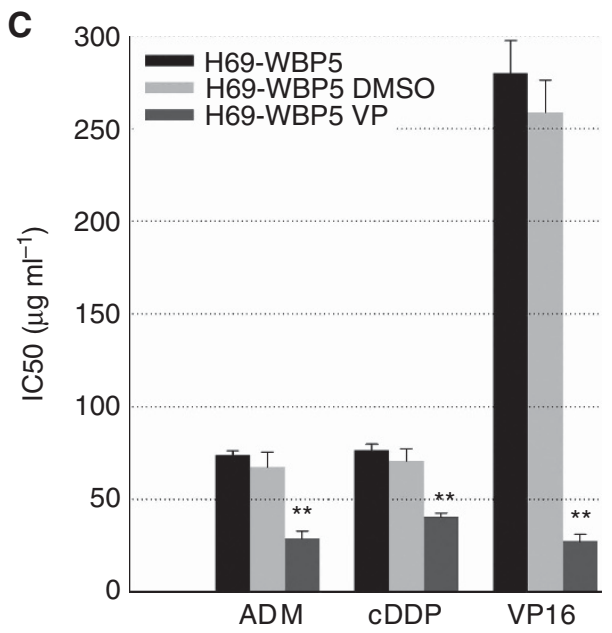

B
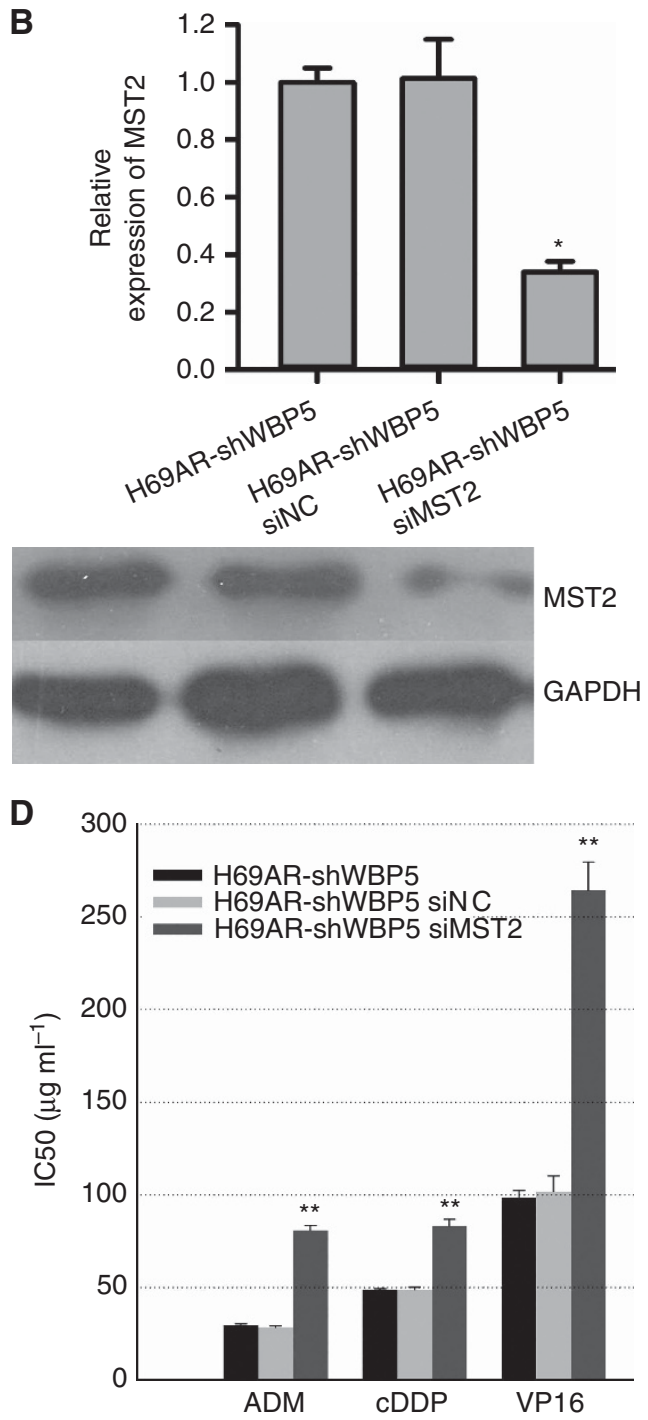

Figure 5. Targeting the Hippo pathway can changeover the MDR of SCLC mediated by WBP5. (A) Schematic representation of proposed mechanism of WBP5-mediated regulation of MDR is under the regulation of miR-335 through the Hippo pathway. (B) MST2 was knocked down by siMST2 both in mRNA and protein level. (C) Accelerating the sequestration of YAP1 in cytoplasm by VP (Verteporfin) could decrease the IC Fo $_{5}$ H69-WBP5; DMSO was used as negative control. (D) Enhancing the nuclear location of YAP1 by knocking down MST2 could increase the IC 50 of H69AR-shWBP5. ${ }^{\star} P<0.05$, ${ }^{*} P<<0.01$, compared with control. 

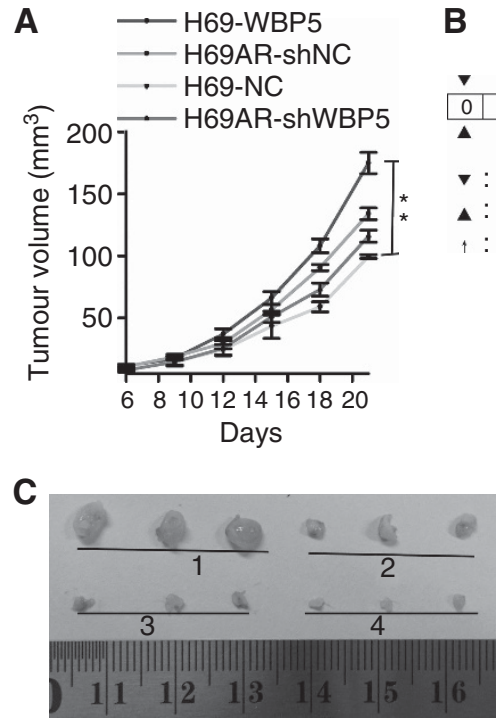

B

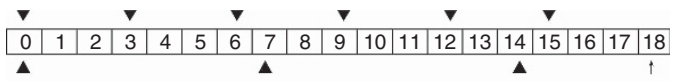

$\checkmark: \operatorname{ADM}\left(2 \mathrm{mg} \mathrm{kg}^{-1}\right)+\mathrm{VP} 16\left(5 \mathrm{mg} \mathrm{kg}^{-1}\right)$

: $\mathrm{cDDP}\left(2 \mathrm{mg} \mathrm{kg}^{-1}\right)$

$\uparrow:$ The mice were euthanised

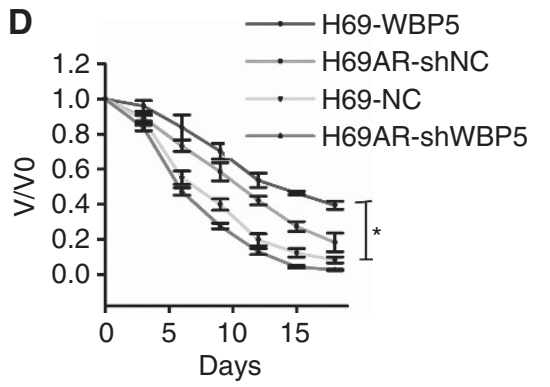

Figure 6. WBP5 can induce SCLC MDR in vivo. (A) Enhancing the expression of WBP5 could increase the tumour growth, whereas reducing the expression of WBP5 could decrease the tumour growth in vivo. (B) Schematic representation of pattern of drugs administration. (C) Tumour xenografts after administration of drugs (1:H69-WBP5; 2:H69AR-shWBP5; 3:H69-NC; 4:H69AR-shWBP5). (D) WBP5 can induce SCLC MDR in vivo. ${ }^{\star} P<0.05,{ }^{\star *} P<0.01$, compared with control.

69AR-shNC) were injected subcutaneously into the flanks of mice. The result showed that upregulation of WBP5 can enhance SCLC tumour growth in vivo $(P<0.01)$, whereas downregulation of WBP5 can decrease SCLC tumour growth (no statistical difference) (Figure 6A).

When the tumours had reached an average volume of about $150 \mathrm{~mm}^{3}$, the mice were treated with Adriamycin (ADM), cisplatin (cDDP) and Etoposide (VP16) (Figure 6B). The tumours formed from H69-WBP5 and H69AR-shNC declined slower than tumours formed from H69-NC and H69AR-shWBP5 after $\mathrm{ADM}+\mathrm{cDDP}+\mathrm{VP} 16$ administration (H69-WBP5 VS H69-NC $(P<0.05)$; H69AR-shWBP5 VS H69AR-shNC (no statistical difference)) (Figure 6C and D).

These findings suggested that WBP5 can promote tumour growth and induce SCLC MDR in vivo.

\section{DISCUSSION}

Some studies have showed that WBP5 is 1 of the 15 candidate oncogenes in human colorectal cancers with microsatellite instability and its expression is associated with advanced gastric cancer with lymph node metastasis (Gylfe et al, 2013; Suh et al, 2015). To the best of our knowledge, the clinical features of WBP5 expression in SCLC have not been reported. In this study, we first analysed 62 cases of SCLC samples and found that WBP5 protein expression is significantly associated with the shorter survival time and clinical stage of the SCLC patients. Cox regression analysis showed that WBP5 was an independent predictor of survival. These data suggested that WBP5 may be an independent predictive indicator of SCLC.

To further validate the biological function of WBP5 in SCLC, we established stable WBP5-overexpressed cells (H69-WBP5 and H446-WBP5) and WBP5-underexpressed cells (H69AR-shWBP5 and H446AR-shWBP5). CCK-8 and flow cytometric analysis indicated that WBP5 can induce MDR by inhibiting apoptosis of SCLC. Tumour xenograft experiments showed that WBP5 can enhance SCLC tumour growth and induce MDR in vivo. Those results suggested that WBP5 may have an important role in growth and MDR of SCLC.
Our previous research has shown miR-335 underexpression and WBP5 overexpression based on genome-wide gene expression profiling analyses in SCLC multidrug resistant cells H69AR compared with parental H69 cells (unpublished data). Upregulation of miR-335 by miR-335 mimics could enhance the drug sensitivity of SCLC cells. In contrast, downregulation of miR-335 using miR-335 inhibitor could induce the MDR. Using PicTar, TarScan and miRBase database, miR-335 was predicted to have potential interaction sites in the $3^{\prime} \mathrm{UTR}$ of WBP5 mRNA. qRTPCR and western blot showed that miR-335 could negatively regulate WBP5. We further confirmed the interaction between miR-335 and WBP5 by dual luciferase reporter assays. According to those data, we concluded that miR-335 may be involved in SCLC MDR by binding to the $3^{\prime} \mathrm{UTR}$ of WBP5.

WBP5 has been reported to bind to Abl, which may be a novel upstream activator of MST2 in the Hippo pathway. We hypothesised that WBP5 may induce SCLC MDR through the WBP5-Abl-MST2YAP1 pathway. Autophosphorylation of MST2 at Thr180 (MST1 at Thr183) is critical for MST1/2 kinase activity (Deng et al, 2003; Praskova et al, 2004). We then detected whether the expression of pMST2-T180 is changed when WBP5 is upregulated or downregulated. The results showed that upregulation of WBP5 can inhibit the phosphorylation of MST2 (pMST2-T180) and YAP1 (pYAP1-S127), thus inducing the nuclear accumulation of YAP1. A similar result was observed when WBP5 was downregulated. These findings demonstrate that WBP5 is involved in the Hippo pathway.

To further confirm whether WBP5 modulates the MDR of SCLC by YAP1 activation, we performed loss-of-function study using YAP1 inhibitor (VP) (Perra et al, 2014). The result showed that the inhibition of YAP1 by VP could blunt the MDR effects of WBP5. We also performed a gain-of-function study by knocking down MST2; the drug sensitivity was turned over. These data reveal that WBP5 modulates the MDR by Hippo pathway.

In conclusion, our study revealed that WBP5 expression is correlated with clinical stage and survival in SCLC patients; we first reported that WBP5 may be an independent predictive indicator of SCLC. This study provides a mechanism of MDR mediated by WBP5 through the Hippo pathway under the regulation of miR335, suggesting that WBP5 may be a candidate target for developing therapeutic strategy to overcome MDR of SCLC. 


\section{ACKNOWLEDGEMENTS}

This work was supported in part by the National Natural Science Foundation of China (81372508). The help and advice of all members of the clinical laboratory center of Zhujiang Hospital is gratefully acknowledged.

\section{CONFLICT OF INTEREST}

The authors declare no conflict of interest.

\section{REFERENCES}

Bai Y, Sun Y, Peng J, Liao H, Gao H, Guo Y, Guo L (2014) Overexpression of secretagogin inhibits cell apoptosis and induces chemoresistance in small cell lung cancer under the regulation of miR-494. Oncotarget 5(17): 7760-7775.

Basu S, Totty NF, Irwin MS, Sudol M, Downward J (2003) Akt phosphorylates the Yes-associated protein, YAP, to induce interaction with 14-3-3 and attenuation of p73-mediated apoptosis. Mol Cell 11(1): 11-23.

Bedford MT, Chan DC, Leder P (1997) FBP WW domains and the Abl SH3 domain bind to a specific class of proline-rich ligands. EMBO J 16(9): 2376-2383.

Bora-Singhal N, Nguyen J, Schaal C, Perumal D, Singh S, Coppola D, Chellappan S (2015) YAP1 regulates OCT4 activity and SOX2 expression to facilitate self-renewal and vascular mimicry of stem-like cells. Stem Cells 33(6): 1705-1718

Bork P, Sudol M (1994) The WW domain: a signalling site in dystrophin? Trends Biochem Sci 19(12): 531-533.

Deng Y, Pang A, Wang JH (2003) Regulation of mammalian STE20-like kinase 2 (MST2) by protein phosphorylation/dephosphorylation and proteolysis. J Biol Chem 278(14): 11760-11767.

Gong M, Ma J, Guillemette R, Zhou M, Yang Y, Yang Y, Hock JM, Yu X (2014) miR-335 inhibits small cell lung cancer bone metastases via IGF-IR and RANKL pathways. Mol Cancer Res 12(1): 101-110.

Guo L, Liu Y, Bai Y, Sun Y, Xiao F, Guo Y (2010) Gene expression profiling of drug-resistant small cell lung cancer cells by combining microRNA and cDNA expression analysis. Eur J Cancer 46(9): 1692-1702.

Gylfe AE, Kondelin J, Turunen M, Ristolainen H, Katainen R, Pitkanen E, Kaasinen E, Rantanen V, Tanskanen T, Varjosalo M, Lehtonen H, Palin K, Taipale M, Taipale J, Renkonen-Sinisalo L, Jarvinen H, Bohm J, Mecklin JP, Ristimaki A, Kilpivaara O, Tuupanen S, Karhu A, Vahteristo P, Aaltonen LA (2013) Identification of candidate oncogenes in human colorectal cancers with microsatellite instability. Gastroenterology 145(3): 540-3 e22.

Haskins JW, Nguyen DX, Stern DF (2014) Neuregulin 1-activated ERBB4 interacts with YAP to induce Hippo pathway target genes and promote cell migration. Sci Signal 7(355): ra116.

Hillgenberg M, Schlehofer JR, von Knebel Doeberitz M, Klein-Bauernschmitt P (1999) Enhanced sensitivity of small cell lung cancer cell lines to cisplatin and etoposide after infection with adeno-associated virus type 2. Eur J Cancer 35(1): 106-110.

Hummel R, Hussey DJ, Haier J (2010) MicroRNAs: predictors and modifiers of chemo- and radiotherapy in different tumour types. Eur J Cancer 46(2): 298-311.

Jafri SH, Glass J, Shi R, Zhang S, Prince M, Kleiner-Hancock H (2010) Thymoquinone and cisplatin as a therapeutic combination in lung cancer: In vitro and in vivo. J Exp Clin Cancer Res 29: 87.

Johnson R, Halder G (2014) The two faces of Hippo: targeting the Hippo pathway for regenerative medicine and cancer treatment. Nat Rev Drug Discov 13(1): 63-79.

Kim Y, Kim H, Park D, Jeoung D (2015) miR-335 targets SIAH2 and confers sensitivity to anti-cancer drugs by increasing the expression of HDAC3. Mol Cells 38(6): 562-572.

Kodaka M, Hata Y (2015) The mammalian Hippo pathway: regulation and function of YAP1 and TAZ. Cell Mol Life Sci 72(2): 285-306.

Lan $\mathrm{H}$, Lu H, Wang X, Jin H (2015) MicroRNAs as potential biomarkers in cancer: opportunities and challenges. Biomed Res Int 2015: 125094.
Liu-Chittenden Y, Huang B, Shim JS, Chen Q, Lee SJ, Anders RA, Liu JO, Pan D (2012) Genetic and pharmacological disruption of the TEAD-YAP complex suppresses the oncogenic activity of YAP. Genes Dev 26(12): $1300-1305$

Liu W, Wu J, Xiao L, Bai Y, Qu A, Zheng Z, Yuan Z (2012) Regulation of neuronal cell death by c-Abl-Hippo/MST2 signaling pathway. PloS One 7(5): e36562.

Metro G, Duranti S, Fischer MJ, Cappuzzo F, Crino L (2012) Emerging drugs for small cell lung cancer-an update. Expert Opin Emerg Drugs 17(1): 31-36.

Mo JS, Park HW, Guan KL (2014) The Hippo signaling pathway in stem cell biology and cancer. EMBO Rep 15(6): 642-656.

Moroishi T, Hansen CG, Guan KL (2015) The emerging roles of YAP and TAZ in cancer. Nat Rev Cancer 15(2): 73-79.

Nagy-Mignotte H, Guillem P, Vignoud L, Coudurier M, Vesin A, Bonneterre V, Toffart AC, Sakhri L, Brambilla C, Brambilla E, Timsit JF, Moro-Sibilot D. Multidisciplinary Thoracic Oncology G (2012) Outcomes in recurrent small-cell lung cancer after one to four chemotherapy lines: a retrospective study of 300 patients. Lung Cancer 78(1): 112-120.

Nemati F, Livartowski A, De Cremoux P, Bourgeois Y, Arvelo F, Pouillart P, Poupon MF (2000) Distinctive potentiating effects of cisplatin and/or ifosfamide combined with etoposide in human small cell lung carcinoma xenografts. Clin Cancer Res 6(5): 2075-2086.

Perra A, Kowalik MA, Ghiso E, Ledda-Columbano GM, Di Tommaso L, Angioni MM, Raschioni C, Testore E, Roncalli M, Giordano S, Columbano A (2014) YAP activation is an early event and a potential therapeutic target in liver cancer development. J Hepatol 61(5): 1088-1096.

Praskova M, Khoklatchev A, Ortiz-Vega S, Avruch J (2004) Regulation of the MST1 kinase by autophosphorylation, by the growth inhibitory proteins, RASSF1 and NORE1, and by Ras. Biochem J 381(Pt 2): 453-462.

Reinke LF, Feemster LC, Backhus LM, Gylys-Colwell I, Au DH (2016) Assessment and management of symptoms for outpatients newly diagnosed with lung cancer. Am J Hosp Palliat Care 33(2): 178-183.

Sudol M, Bork P, Einbond A, Kastury K, Druck T, Negrini M, Huebner K, Lehman D (1995a) Characterization of the mammalian YAP (Yesassociated protein) gene and its role in defining a novel protein module, the WW domain. J Biol Chem 270(24): 14733-14741.

Sudol M, Chen HI, Bougeret C, Einbond A, Bork P (1995b) Characterization of a novel protein-binding module-the WW domain. FEBS Lett 369(1): $67-71$.

Suh YS, Yu J, Kim BC, Choi B, Han TS, Ahn HS, Kong SH, Lee HJ, Kim WH, Yang HK (2015) Overexpression of plasminogen activator inhibitor-1 in advanced gastric cancer with aggressive lymph node metastasis. Cancer Res Treat 47(4): 718-726.

Takahashi N, Yamamoto E, Ino K, Miyoshi E, Nagasaka T, Kajiyama H, Shibata K, Nawa A, Kikkawa F (2009) High expression of $\mathrm{N}$-acetylglucosaminyltransferase $\mathrm{V}$ in mucinous tumors of the ovary. Oncol Rep 22(5): 1027-1032.

Wang K, Chen X, Zhan Y, Jiang W, Liu X, Wang X, Wu B (2015) miR-335 inhibits the proliferation and invasion of clear cell renal cell carcinoma cells through direct suppression of BCL-W. Tumour Biol 36(9): 6875-6882.

Wu C, Xu B, Yuan P, Miao X, Liu Y, Guan Y, Yu D, Xu J, Zhang T, Shen H, Wu T, Lin D (2010) Genome-wide interrogation identifies YAP1 variants associated with survival of small-cell lung cancer patients. Cancer Res 70(23): 9721-9729.

Zhang AX, Lu FQ, Yang YP, Ren XY, Li ZF, Zhang W (e-pub ahead of print 24 June 2015) MicroRNA-217 overexpression induces drug resistance and invasion of breast cancer cells by targeting PTEN signaling. Cell Biol Int.

Zhao B, Li L, Lei Q, Guan KL (2010) The Hippo-YAP pathway in organ size control and tumorigenesis: an updated version. Genes Dev 24(9): 862-874.

Zhao B, Ye X, Yu J, Li L, Li W, Li S, Yu J, Lin JD, Wang CY, Chinnaiyan AM, Lai ZC, Guan KL (2008) TEAD mediates YAP-dependent gene induction and growth control. Genes Dev 22(14): 1962-1971.

This work is published under the standard license to publish agreement. After 12 months the work will become freely available and the license terms will switch to a Creative Commons AttributionNonCommercial-Share Alike 4.0 Unported License.

Supplementary Information accompanies this paper on British Journal of Cancer website (http://www.nature.com/bjc) 